\title{
Somatoforme Störungen in Humanistischer und Körperpsychotherapie
}

\author{
Jörg Claner
}

\author{
Psychotherapie-Wissenschaft 9 (2) 98-106 2019 \\ www.psychotherapie-wissenschaft.info \\ CC BY-NC-ND \\ https://doi.org/10.30820/1664-9583-2019-2-98
}

\begin{abstract}
Zusammenfassung: Mit der Klage über ausschliesslich körperliche Beschwerden und einer strikten Ablehnung psychosomatischer Erklärungen begegnen uns die als schwierig geltenden PatientInnen mit somatoformen Störungen (SSD). Das Schmerzerleben wird zum Beispiel nur körperlich wahrgenommen und sie finden dafür keinen seelisch-gefühlsmässigen Beziehungskontext (Alexithymie). Ätiologische Überlegungen helfen uns bei einem tieferen Verständnis ihres spezifisch leidvoll-schmerzhaften Körpererlebens und ihrer Beziehungsformen. SSD-PatientInnen haben meist keine liebevolle, körperlich-emotionale und seelische Resonanz bei frühen Bezugspersonen erlebt und dabei gelernt, ihren Körper als (dysfunktionales) Objekt zu betrachten. Körperpsychotherapie kann diesen Symptomfokus gut aufgreifen. Es werden Prinzipien und Möglichkeiten einer differenzierten Förderung der Körperwahrnehmung als Basis für hilfreiche Veränderungen bei SSD beschrieben - und soweit vorhanden, entwicklungspsychologische und neurobiologische Zusammenhänge ausgeführt. Die neue Selbstwahrnehmung sowie erklärenden Informationen verhelfen Betroffenen zu einem selbstbestimmteren/wirksameren Umgang mit ihren Störungen und zu neuen Möglichkeiten, Gefühle für die Gestaltung von Beziehungen und ihres Lebens zu nutzen. Vorhandene Studien sprechen dafür, dass ein Vorgehen mit den hier beschriebenen Grundprinzipien hilfreich sein kann.
\end{abstract}

Schlüsselwörter: Somatoforme Störung, Schmerzerleben, Alexithymie, Körperschemastörung, Familiäre Kooperation, Körperpsychotherapie, Körperwahrnehmungsschulung

\section{Einleitung}

Der leitende Internist einer neurologischen Fachklinik hielt 1995 triumphierend eine internistische Fachzeitschrift hoch. In einem Artikel wurden Nachweis und Bedeutung von «Helicobacter pylori» für die Entstehung von Magengeschwüren beschrieben (vgl. Leiß, 2011). Er dozierte anschliessend über den «Unsinn der ganzen Psycho-Vorstellungen». Viele PatientInnen mit Somatoformen Störungen (SSD) haben vergleichbare Wünsche: Es soll endlich eine kompetente medizinische Fachkraft die körperliche Ursache ihres Leidens finden und beseitigen. Eine Motivation für eine Behandlung, die Emotionen, sozialen Beziehungen und der Lebensgeschichte eine einflussreiche Bedeutung beimisst, ist erst einmal nicht gegeben.

Im Denken westlicher Kulturen herrscht seit Jahrhunderten eine Trennung von Körper und Seele vor. Erstaunlicherweise halten viele Fachleute des Gesundheitswesens immer noch an dieser Trennung fest. Es ist noch längst kein fachliches Allgemeingut, dass bei jeder Erkrankung bio-psycho-soziale Faktoren eine Rolle spielen (Egger, 2017; v. Uexküll et al., 1997). Daher finden sich solche Ansichten auch in der Bevölkerung, in der Menschen nicht selten Psychisches mit «Verrückt-sein» oder «eigenem Versagen» assoziieren und psychosomatische Erklärungen als kränkend erleben. Bei PatientInnen mit SSD scheint dies nach unserer Erfahrung in besonderem Masse der Fall (vgl. Rudolf, 2008).
Für medizinische wie psychotherapeutische Behandelnde ist es elementar, um das beschriebene Spannungsfeld zu wissen und PatientInnen kooperativ zu behandeln (Egger, 2017; Hennigsen et al., 2006). Für Humanistische PsychotherapeutInnen ist es selbstverständlich, dass somatische, psychische, interpersonelle sowie soziokulturelle Perspektiven miteinander verwoben sind.

\section{SSD - eine Standortbestimmung}

Das diagnostische Manual DSM-V spricht inzwischen von «Somatic Symptom and related Disorders (SSD)» für PatientInnen, die über «Somatische Belastungsstörungen mit ausschließlich körperlichen Beschwerden» klagen, und unterscheidet nicht mehr zwischen «somatoformen", also bisher medizinisch nicht erklärlichen Symptomen sowie andererseits körperlichen Beschwerden, bei denen die vorhandenen organpathologischen Veränderungen allein das gesamte Krankheitsgeschehen nicht hinreichend erklären (vgl. Lahmann et al., 2016). Die alte Unterscheidung wurde so zwar aufgegeben, im Hintergrund existiert das offenbar schwer lösbare Spannungsfeld aber weiter: a) Es gibt zum einen die «Somatoformen Störungen» obne nachweisbare organische Erkrankung, wie die "Somatic Symptom Disorders», «Illness Anxiety Disorders», "Conversion Disorders» (und weitere Untergruppen). Sie werden von MedizinerInnen weiter bevorzugt als funktionelle Störungen betrachtet, wur- 
den vormals psychodynamisch als Organneurosen auf der Basis von emotionalen Entwicklungsdefiziten und Selbstwertstörungen angesehen (vgl. Ermann, 1997).

b) Daneben beschreibt die Kategorie «Psychological Factors Affecting other Medical Conditions» die ehemaligen «Psychosomatosen» wie Asthma oder entzündliche Darmerkrankungen, so wie es im ICD-10 mithilfe der Kategorisierung F54 geschah. Alexander (1977) hatte ehemals diese Psychosomatosen mit vorhandenen Organdestruktionen beschrieben und auf seelische Verursachungen zurückzuführen versucht.

Beide Gruppen werden nun unter SSD kategorisiert! PsychotherapeutInnen sollten bedenken, dass das DSM ein System vornehmlich amerikanischer PsychiaterInnen ist, mit einer der Fachgruppe immanenten, eher biologischen Perspektive. Psychotherapeutischen KlinikerInnen lag der ICD-11 der WHO bisher nicht vor. In ihm wird diese Erkrankungsgruppe als «Bodily Distress Disorder (BDD)» beschrieben, mit einer Multi-Organ und einer Single-Organ-Variante. Die aktuelle S3-Leitlinie spricht abweichend von «nicht-spezifischen, funktionellen und somatoformen Körperbeschwerden» (Schaefert et al., 2012).

Der Umgang mit dieser recht heterogenen PatientInnengruppe gilt als schwierig und undankbar. Die neuen Klassifikationen versuchen dezidiert, die zuvor beklagte Stigmatisierung mit den bisherigen Diagnosesystemen $z u$ vermeiden. Das zentrale Merkmal ist nun lediglich das Vorhandensein körperlicher Symptome, die mit einer klinisch bedeutsamen Belastung durch die Beschwerden und erheblichem subjektiven Leiden einhergehen. Zur Vereinfachung werden wir von «SSD» sprechen und nicht mehr, wie früher bei KlinikerInnen üblich, von Psychosomatischen Krankheiten.

\section{Komorbidität bei SSD-PatientInnen}

Die Selbstwahrnehmung von SSD-PatientInnen ist oft von grosser Angst (bis zu 60 \%; Morschitzky, 2007) gefärbt und auf die körperlichen Beschwerden im eigenen Körperselbst eingeengt. Diese Fixierung kann zu einer Verstärkung der Symptomatik führen. Fehlt ein sinnvoller Bezug zwischen körperlicher Empfindung und deren emotionaler Bedeutung, so kann kein emotionaler und sozialer Verarbeitungsprozess gelingen. Dann machen sich leichter Hilflosigkeit, Ohnmacht, Verzweiflung und sozialer Rückzug breit, es findet sich Depression in bis zu $90 \%$ der Fälle (ebd.), was wiederum die Schmerzwahrnehmung steigert. Ob die Depression sekundär entsteht oder als depressive Somatisierung mitursächlich ist, bleibt offen. In einem sinnvoll verstandenen bio-psycho-sozialen Krankheitsmodell macht ein Streit darüber auch keinen Sinn (Egger, 2017, S. 33f.). Rudolf (2008) nahm bei SSD einen depressiven Grundkonflikt mit dysfunktionaler Bewältigung an, was zu lieblosen, nicht spiegelnden Eltern passen würde, und Morschitzky (2007) schreibt von einer Komorbidität von Persönlichkeitsstörungen von bis $45 \%$.

\section{Erklärungsmodelle}

\section{Defizit-Modelle der Selbstentwicklung}

Im Sinne eines Defizit-Modells der Entwicklung gehen wir, wie viele KollegInnen (Hennigsen et al., 2006; Rudolf, 2008; Röhricht, 2011a) von einer frühen Störung der Selbstentwicklung aus. Bei dieser wurde das Körper(kern)selbst (Stern, 1992) nicht mit Emotionen sowie einer Versprachlichung und Mentalisierung der Emotionen und des Beziehungserlebens verknüpft. Das Körperkernselbst bildet die Grundlage des Entwicklungsmodells des Selbsterlebens bei Stern (ebd.). Für seine Ausbildung ist eine störungsfreie sensomotorische Integration unserer Körpersignale im Gehirn zu einem Gesamtbild von unserem "Selbst» erforderlich (vgl. Ayres, 2002; Blakeslee \& Blakeslee, 2007, v. Uexküll et al., 1997). Sensomotorische Dialoge mit frühen Betreuungspersonen hat Trevarthen (2009) als Protokonversationen bezeichnet. Zusammen mit Berührung bilden sie die Grundlage der menschlichen Entwicklung (LaPierre, 2003; Linden, 2015; Panagiotopoulou et al., 2017). Über Mimik und Körperhaltungen werden aus der familiären Atmosphäre affektmotorische Haltungsmuster, viszerale Gefühle und visuelle Bildeindrücke übernommen, die nie ins explizite Bewusstsein treten müssen. Das kooperative Zusammenspiel von Bewegungen und Rhythmen ist die Basis für das, was wir wahrnehmen und die Grundlage für die späteren Interpretationen auf den abstrakteren Ebenen von Psyche und Denken. Es lässt das «Implicit Relational Knowing» (Stern et al., 2012; Fuchs \& De Jaegher, 2010) entstehen. Dies implizite Wissen oder Leibgedächtnis bei Fuchs (2009) wird dabei als der eigentliche Träger unserer Lebensgeschichte, unserer persönlichen Identität betrachtet. Die frühen Erfahrungen von Rhythmen, Verbundenheit und Freude oder aber von Schreck und Schock - vor allem in den ersten zwei Lebensjahren - bleiben besonders in körperlichen Haltungs- und/oder Bewegungsmustern (Mikropraktiken bei Downing, 2006, 2007) im implizitprozeduralen, vornehmlich rechtshirnigen Gedächtnis sowie in überdauernden Veränderungen der Regulation von Emotionen und vegetativen Funktionen durch das Autonome Nervensystem (ANS) und Limbisches System erhalten (Schore, 2005, 2011).

Macht das Kleinkind/Baby emotional beruhigende Erfahrungen der Zuwendung und Affektabstimmung in der Beziehung zu Betreuungspersonen, so erlebt es eine Neurozeption von Sicherheit mit Aktivierung des prosozialen ventralen Vagus-Systems (VVC) (Porges, 2010) und bei Wiederholungen eine sichere Bindung (Beebe \& Lachmann, 2004). Diese ist verbunden mit dem Aufbau eines gesunden, stabilen körperlichen Selbstbezugs und einer entsprechenden emotionalen Selbstregulation. Fehlt eine solche Erfahrung - aus welchen Gründen auch immer -, so werden sich die körperlich-emotionale Erregung (Stress) und damit auch die Beschwerden verstärken und bei der Wiederholung solcher Erfahrungen besonders vor dem dritten Lebensjahr im implizit-prozeduralen (Leib-) 
Gedächtnis verankern. Wenn das Kind mit seinen körperlich erlebten Beschwerden und Ängsten allein bleibt, wird es auf diese zurückgeworfen. Die körperlichen Missempfindungen bleiben dann ohne Gefühlsbezug und Sprache - sowohl im Kontakt zum Gegenüber als auch zu sich selbst. Dies finden wir später bei PatientInnen mit SSD.

Gibt es wiederholt hilfreiche emotionale Beziehungserfahrungen, so verbinden sich diese später mit Symbolen, Sprache und Mentalisierungen. So wird mit der Zeit der rechte präfrontale Cortex in die Lage versetzt, eine Modulierung der Emotionsverarbeitung besonders im Mandelkern/Amygdala zu bewirken. Dies kann zum Beispiel zur Dämpfung des Stresses einer sympathikotonen Erregung beitragen (Rudolf, 2008). Diese sich erst spät entwickelnde, übergeordnet steuernde, präzentrale Grosshirnfunktion ist im Falle von Traumatisierungen (auch Verlassenheit) oder schweren Beeinträchtigungen nur eingeschränkt wirksam und scheint bei SSD unzureichend ausgebildet zu sein. Die kognitive und verbale Steuerung ist dann eingeschränkt (Bauer, 2011; Schnabel, 2019).

Soziale Ausgrenzung und Ungerechtigkeit ist in jeder Lebensphase ausgesprochen schmerzhaft, es aktiviert im Gehirn die gleichen Areale wie körperlicher Schmerz (Eisenberger et al., 2003, 2006; Bauer, 2011). Zudem dient Schmerz in der Erziehung oft als Hilfsmittel der Disziplinierung (Fuchs, 2009). Die begleitenden negativen Emotionen verstärken dabei das Schmerzerleben, das andererseits durch Berührung und eigenes Kontrollerleben in der Situation gedämpft wird. Berührung kommt zudem eine zentrale Funktion für die Ausbildung von Selbstempfinden, Selbststeuerung und auch Bindung zu; interpersonale emotionsgetragene Berührung fördert Vertrauen und kooperatives Verhalten (Linden, 2015; LaPierre, 2003; Panagiotopoulou et al., 2017; Porges, 2010). Berührung bedarf aber einer besonderen Achtsamkeit. Bedrohliche oder traumatisierende Erfahrungen in der Lebensgeschichte machte zum Beispiel bei einem Crohn-Patienten Berührung in der Psychotherapie problematisch. In einem solchen Fall kann aber die Erforschung und Bearbeitung dieser Probleme Zugang zu wichtigen Lösungsansätzen bieten (Clauer, 2008, 2014).

\section{Ätiologie I: Frühe Defizite}

Störungen in der seelischen und körperlichen Entwicklung können Kinder als repetitive Beeinträchtigungen oder frühe Traumatisierungen (wie z.B. Nicht-Beachtung, Feindseligkeit, Verlassenheit, aggressive Gewalt, sexueller Missbrauch oder emotionale Vernachlässigung) erlitten haben. Als Bindungswesen können wir aber nicht anders, als auch diejenigen zu lieben, zu imitieren und zu verinnerlichen, die uns misshandeln. Von alledem wissen SSDPatientInnen und ihre Familien oft nichts (mehr), oder aber der familiäre Kontext will oder darf davon nichts wissen. Die Klage der PatientInnen konzentriert sich ausschliesslich auf den Körper, auf die Ebene der aktuellen sensomotorischen Sensationen (die Empfindungsebene), und nicht auf die emotionale Ebene! Mit Sensationen sind hier Empfindungen wie Lust/Unlust, Schmerz, Wärme/ Kälte, Spannungen, Steifigkeit etc. gemeint - im Unterschied zu Affekten und Gefühlen. PatientInnen erleben sich und den Körper als zwei gespaltene Entitäten, sehen den Körper als defizitäres und reparaturbedürftiges Objekt. Die Beschäftigung mit der Psyche und emotionalem Erleben in Beziehungen wäre für sie so, als ob ihre Person defizitär und sie daher verrückt wären. Die Verknüpfung mit Emotionen und sozialen Faktoren (ausser Schmerz) wird folgerichtig nicht gesehen oder gar abgestritten, aus therapeutischer Perspektive also verleugnet oder abgespalten.

Affekte und Emotionen sind immer mit Körperreaktionen verbunden und sind eine Bewusstwerdung körperlicher Erregungszustände. Die Körperwahrnehmung ist damit Übermittler von Affekten, beide gehören untrennbar zusammen. Die Förderung der Körperwahrnehmung ist aus unserer Sicht daher die grundlegende Basis für eine Veränderung bei SSD-PatientInnen. Dann können sie erfahren, dass Affekte über den Stand der Interaktionen mit der Umwelt informieren und helfen können, mit übermässiger Erregung und Belastung umzugehen.

Aus lerntheoretischer Sicht kann man annehmen, dass die Umwelt nur mit körpersymptombezogenen Klagen und Protesten im Körperausdruck zu erreichen oder zumindest zur Aufmerksamkeit im Sinne einer funktionalen Versorgung/Behandlung zu bewegen war. Ein (direkter) Ausdruck von aversiven Emotionen und der Protest über Stimme und Gesten fand wohl keine Beachtung und Akzeptanz und kann dann später nicht differenzierend mit Symbolen/Sprache verknüpft werden. Die Annahme des «NEIN», des Protestes ist aber entscheidend für die aufeinander aufbauenden Stufen der Selbstentwicklung (Clauer, 2014).

In einer bio-psycho-sozialen Sichtweise sind die unter Belastung auftretenden Körpersymptome als bestmögliche Anpassungsleistung/organismische Selbstregulation (aber eben auch dysfunktionale Notlösung) zu verstehen. Wächst das kleine Kind in einer Umgebung auf, in der es sich verlassen, erschreckt und verängstigt, bedroht oder gar gequält und ungerecht behandelt fühlt, so passt es sich auch an diese Umgebung an. Der Organismus hat in seiner Beziehung zur Umwelt und zu sich keine anderen Regulationsmöglichkeiten gelernt. Der Körper wird/bleibt so das alleinige Ausdrucksorgan innerer Vorgänge. Ein sich ergebendes Gefangensein im eigenen Körper-Unbehagen kann auch bei SSD als eine Variante der Körperschema-/Körperbild-Störungen verstanden werden (Henningsen, 2007).

\section{Ätiologie II: Frühe Beziehungskontexte bei SSD}

Auf der Ich-Du-Ebene geht es den PatientInnen darum, das körperliche Mangelerleben (Schmerz, subjektive Dysfunktion) von aussen als objektivierte Dysfunktion bestätigt zu bekommen. Es gibt im Kontakt zur/m The- 
rapeutin/en keine Ich-Du-Aussagen, der Körper wird als Objekt dazwischen geschoben. Der eigene Körper wird zum Feind erklärt, der das Opfergefühl auslöst. Im Umkehrschluss könnte das bedeuten, dass die PatientInnen in ihrer frühen Entwicklung die Erfahrung gemacht haben, dass sie von Eltern mit (körper-)feindlichen Haltungen und Impulsen «behandelt» wurden (vgl. Rudolf, 2008). Sie wurden vermutlich in ihrem Körper von den Eltern nicht freundlich angeschaut, nicht zärtlich berührt, nicht rhythmisch bewegt und getragen, sodass es grundlegende Spiegelungsdefizite in der frühen Kindheit gab. Besonders eingeschränkte rhythmische sensomotorische Abstimmung und positiver emotionaler Blick- und Körperkontakt/Beziehungsregulierung sowie Verleugnungen von Problemen und der Mangel an Verbalisierungen führen zu einer Behinderung der Entwicklung der Gefühlsfähigkeit und des Spürens von Stimmigkeit (vgl. Längle, 2009, S. 21).

So gesehen kann der objekthafte Blick auf den eigenen Körper als ein elterliches, negatives Introjekt verstanden werden - ohne Bewusstsein von den früheren Beziehungskontexten in der vorsprachlichen Zeit. In diesem Sinne verstehen wir hier Introjekte als körperlich eindringliche, «implantierte» (unbewusste) Haltungen der Eltern dem kindlichen Körper gegenüber. Die/der PatientIn «behandelt» sich nun selbst so, wie es die Bezugspersonen früher taten. Prinzipiell waren es Grenzverletzungen, Störungen der Selbstregulation des Organismus, die zu einer mangelhaften Entwicklung von selbstregulativen Fähigkeiten im Kontext einer interaktiven Regulation führen (vgl. Beebe \& Lachmann, 2004).

\section{SSD und die Kooperation im Familienrahmen - eine systemische Perspektive}

Wir beobachten bei SSD-PatientInnen einen grossen Drang zur Kontrolle über Körper, Umwelt und Beziehungspartner (wie Behandelnde) und entdecken oft eine in ihrer Lebensgeschichte vorhandene Parentifizierung, bei der sie für die Eltern sorgten, was eine ausgesprochene altruistische Leistungsbereitschaft auf den Weg bringen kann. Die bisher angeführten Erklärungsmodelle wie depressiver Grundkonflikt oder strukturelle Defizite leiten sich aus einem dyadischen Beziehungsverständnis und den entsprechenden Mutter-Säuglings-Untersuchungen ab. In der Entwicklung bilden jedoch Mutter und Vater oder mehrere Bezugspersonen das körperliche und emotionale Universum des Babys.

Die verzweifelte Suche der SSD-PatientInnen nach Resonanz und Verständnis statt Kritik und Ablehnung erinnert an Kinder, die nacheinander versuchen, sämtliche Familienmitglieder auf etwas aufmerksam zu machen, was (lebens-)bedrohlich mit ihnen nicht in Ordnung ist. Systemisch gesehen könnte es sich - im Sinne der Triangulierung - auch um eine Re-Inszenierung von Erfahrungen mit untereinander uneinigen oder feindselig konkurrierenden Eltern handeln, die wir häufig in der Anamnese der PatientInnen finden (Clauer, 2014).
Für die therapeutische Arbeit mit SSD-PatientInnen lohnt es sich, die systemische Sicht zur Entwicklung von Familienallianzen im «Primären Dreieck» (aus Mutter, Baby und Vater) kurz zu betrachten (Fivaz-Depeursinge \& Carboz-Warnery, 2001; v. Klitzing, 2002; Clauer, 2009a). Von der Kooperationsfähigkeit der Eltern hängt es ab, ob sie die Affektabstimmung bei unvermeidbaren Abstimmungsunterbrechungen, zum Beispiel in Spielsituationen, wiederherstellen können. Sind sie feinfühlig und zur Kooperation miteinander fähig, können sie die affektmotorischen und -sensorischen Krisen des Babys auffangen, halten und aushalten (containen), und ihrem Kind so einen entwicklungsförderlichen Rahmen bereitstellen. Diese rahmenden und kooperativen Fähigkeiten der Eltern sind bei dysfunktionalen Familienallianzen nur unzureichend vorhanden. In der Untergruppe der sogenannten kollusiven Familienallianzen mit umleitenden Triangulierungen hat das dabei parentifizierte Kind von Anfang an die Aufgabe, Hüter der Einheit des Elternpaares zu sein, um den Zerfall der Bezogenheit zu Dritt zu verhindern, anstatt von den Eltern emotionalen Rückhalt zu erhalten. Dies begünstigt einen vermeidend-kontrollierenden Beziehungsstil, wie wir ihn oft auch bei SSD-PatientInnen finden. Für den Nachweis einer Bedeutung solcher Lebenserfahrungsmuster in der Geschichte von SSD-PatientInnen wären allerdings Längsschnittuntersuchungen bei Kindern aus Familien mit solchen kollusiven Kooperationsmustern der Eltern erforderlich. In einer ähnlich kollusiv-konflikthaften Kooperation finden sich später oft auch die Behandelnden von SSD-PatientInnen wieder und werden leicht in eine solche Dynamik hineingezogen.

\section{Folgen von Entwicklungsdefiziten und Traumata: Gestörtes Körperempfinden und Gefühlsblindheit (Alexithymie)}

Affekte und Emotionen sind eine Bewusstwerdung körperlicher (Erregungs-)zustände und immer mit Körperreaktionen verbunden. Gefühle erleben wir so im Körper, quasi als interpretierte Körperempfindungen. Jedes Gefühl ist mit einem Strauss mehr oder weniger spezifischer Reaktionsmuster des Körpers verbunden, Angst zum Beispiel mit Enge in Brust und Hals, den Blutgefässen (hoher Blutdruck) und muskulärer Anspannung. Der Wahrnehmungsprozess solcher Reaktionen und von Affekten beginnt der Hirnforschung zufolge (Damasio, 2011; vgl. Clauer, 2014) mit einer Bewusstseinsbildung schon im Hirnstamm, und zwar vor allem der enterozeptiven Signale aus dem Bereich von Hals, Brust und Bauch. Gerade diese Bereiche sind es, in denen viele SSD-PatientInnen ihre Beschwerden erleben (z. B. Magen-Darmbeschwerden). Bei ihnen ist die Fähigkeit, Gefühle wahrzunehmen, zu identifizieren und auszudrücken eingeschränkt funktionsfähig oder blockiert (vgl. z. B. Traue, 1998). Sie können nicht nur eigene Gefühle schwer wahrnehmen, sondern haben auch Defizite in der Erkennung emotionaler Gesichtsausdrücke (Pedrosa et al., 2008). Das Nicht-Lesen-Können von Gefühlen wird als Alexithymie (auch Seelenblindheit) bezeichnet. Dabei 
ist wohl die Integration links- und rechtshirniger Prozesse beeinträchtigt (Traue, 1998; Lausberg, 2006, 2017). Ob zu einer solchen Spaltung/Dissoziation auch das Miterleben einer dysfunktionalen Kooperation der Eltern beiträgt, darüber kann hier nur spekuliert werden. In unserer Anamnese von SSD-PatientInnen finden wir häufiger als angegeben eine Vorgeschichte oder Komorbidität mit Essstörungen (Morschitzky, 2007; Lausberg, 2017). Dies wäre naheliegend, da wir in beiden PatientInnengruppen Alexithymie und eine beeinträchtigte Selbstregulation vorfinden.

\section{Wut, Angst, Schmerz und Körperspannungsmuster}

Im Kontext einer Depression bei SSD findet sich häufig eine altruistische Haltung mit einer durchhaltewilligen Leistungsbereitschaft, oder aber narzisstische Züge, bei denen beispielsweise die Heilserwartungen an die (idealisierten) MedizinerInnen bald zu einer intensiven Enttäuschungswut, drängenden Klagen und der Entwertung des Arztes bzw. der Ärztin verbunden mit Beziehungsabbruch führen können (Rudolf, 2008; Röhricht, 2011a). Eine unterdrückte Enttäuschungswut ist für PsychotherapeutInnen ein wichtiger Ansatzpunkt. Seelischer Aufruhr bei enttäuschten Erwartungen oder sozialer Ausgrenzung, wie auch die Angst hinter einer altruistischen Haltung, ist mit sympathikotoner Erregung, hoher innerer Anspannung von Muskeln und Arteriolen und damit Durchblutungsstörungen und schliesslich Erschöpfung der Kortisolspeicher verbunden, bevor es vielleicht zu einem Zusammenbruch des Systems mit den Symptombildungen kommt (vgl. Rudolf, 2008). Viele Menschen kennen Ähnliches in milderer Form als Urlaubsgrippe oder Erkrankung nach einer beruflichen Überforderungsphase.

Seit Beginn der 1950er Jahre ist aus der Psychosomatik-Forschung durch die Arbeiten von Alexander (bspw. 1977) bekannt, dass vor allem unterdrückte Impulse der Selbstbehauptung oder unterdrückte feindselige Impulse direkte physiologische Auswirkungen haben und Ursache somatischer Symptombildung wie zum Beispiel Bluthochdruck sein können, was sich auch als Folge von Angst und Stress betrachten lässt. Seitdem wurde eine Vielzahl derartiger psychosomatischer Zusammenhänge empirisch belegt (z.B. Heinrich, 1986; Traue, 1998; Tschuschke, 2017). Auf die chronischen Auswirkungen auf Organsysteme wurde schon hingewiesen (Clauer, 2014).

Die eingeschränkte Fähigkeit zu Protest und «Nein», also dazu, negative emotionale Inhalte (Ärger, Wut, Frustration, Ekel etc.) zu kommunizieren, wird als ein zentrales Problem bei SSD-PatientInnen beschrieben (Röhricht, 2011a). Emotionen haben eine direkte Verbindung zur Muskulatur des gesamten Körpers, sie haben eine motorisch-expressive Komponente (vgl. Krause, 2006, S. 28). Je nach Qualität des eingeschränkten Gefühlsausdrucks werden verschiedene funktionelle Einheiten des Körpers angespannt und gehalten, die nicht unbedingt im engeren Sinne anatomisch verbunden sind. Jeder Mensch zeigt spezifische Haltungs-, Ausdrucks- und Bewegungs- muster. Die Exploration der Muster kann in der Therapie psychische Prozesse in Gang setzen, die Aufschluss über die zugrundeliegenden impliziten Erinnerungen und emotionalen Erfahrungen geben (Heinrich-Clauer, 2017).

Zum besseren Verständnis geben wir hier ein Beispiel für solche «Lebenshaltungen»: Bei Babys können bedrohliche Erfahrungen mit Bezugspersonen leicht zu Verspannungen und Haltungsstörungen in der Nacken-, Hals- und Kiefermuskulatur führen und chronisch werden. Diese Schutzreaktion gegen eine existenzielle Bedrohung nennt die Bioenergetische Analyse "Cephaler Schock» (Lewis, 2008). OrthopädInnen finden dabei Veränderungen im Halsbereich und besonders im Atlanto-OccipitalGelenk und ZahnärztInnen Zahnveränderungen durch Zähneknirschen. Die Betroffenen können dabei nicht vertrauensvoll loslassen und sich dem Erdboden (oder einer entsprechenden Beziehung) anvertrauen. Sie hängen mit ihrer Lebenshaltung quasi in der Luft, und ihr Stand in der Welt ist entsprechend unsicher. Eine Therapie bei OsteopathInnen und PhysiotherapeutInnen kann neben einer körperpsychotherapeutischen Arbeit hilfreich sein und ist ein gutes Beispiel für die Bedeutung einer Zusammenarbeit zwischen verschiedenen Fachgruppen.

\section{Therapeutische Zugänge zu SSD-PatientInnen}

Grundlage für eine erfolgreiche therapeutische Arbeit ist, dass der SSD-Kranke sich in seinem Leiden und somatischen Krankheitsüberzeugung ernst genommen fühlt. Körperorientiert arbeitende humanistische PsychotherapeutInnen kommen für sie am ehesten infrage (Röhricht et al., 2019). Mit SSD-Betroffenen ist es hilfreich, sie einfühlsam nur soweit zu begleiten und ein Verständnis sowie eine Handhabung ihres Leidens zu erarbeiten, wie sie bereit sind, mitzugehen.

\section{Klinik- und Gruppenbehandlungen}

Behandelnde ÄrztInnen werden oft von den drängenden, sich wiederholenden Behandlungsforderungen an den Rand ihrer Möglichkeiten gebracht, und Psychotherapie erscheint den SSD-Betroffenen als abwegig (Lahmann et al., 2016; Röhricht, 2011a; Rudolf, 2008). Ein möglicher und probater Ausweg stellt eine Gruppenbehandlung in einer Tages- oder Fachklinik dar, die am besten eine Integration von somatischer Behandlung mit der von Stressfolgen, Bewältigung von Überlastungen und Psychoedukation anbieten kann (z. B. Arbeitskreis PISO, 2011).

Die strukturierten Behandlungsprogramme betonen, dass nicht Heilung das vordringliche Ziel ist, sondern das Erlernen eines lindernden, verbesserten Umganges mit den Beschwerden. Solche Programme mit psychoedukativer Unterrichtung kommen der Vermeidung von Schuldthemen und dem Kontrollbedürfnis von SSDPatientInnen entgegen. Sie setzen an der Bewältigung von Stress und Belastungen an, initial mit dem Erlernen und Üben verschiedener Entspannungsverfahren, und 
fördern die Achtsamkeit und Exploration für das eigene Körper- und Gefühlserleben. Dies geschieht häufig mithilfe erlebnisfördernder Methoden der Humanistischen Psychotherapie (HP)(vgl. Eberwein \& Thielen, 2014). Sowohl in Einzel- wie in Gruppentherapie sind Körperwahrnehmung und Körperpsychotherapie (KPT) ein wichtiger und besonders geeigneter Bestandteil, sodass auch eine Manual-gestützte KPT-Gruppentherapie entwickelt wurde (Röhricht, 2011a). Bisherige Ergebnisse zeigen, dass eine körperpsychotherapeutische Behandlung bei SSD erfolgversprechend ist (Nickel et al., 2006; Röhricht et al., 2019).

Die Aktivierung durch gemeinsame körperliche Übungen und Sport, die (ähnlich wie bei depressiven PatientInnen) auch zu Hause weitergeführt werden können, wirkt nicht nur verbreiteten Schonhaltungen und fehlender Vitalität entgegen, sondern kann den PatientInnen neue Erfahrungen ermöglichen, selbst etwas für sich erreichen und bewirken zu können. Auch viele kunsttherapeutische Verfahren wie die Arbeit mit Ton oder Malen können die Selbsterkundung fördern (v. Arnim et al., 2007; Röhricht, 2011b). Sie helfen den PatientInnen, neue Perspektiven auf das Selbsterleben zu entdecken und für Nöte und Konflikte Ausdrucksformen, Symbole und schliesslich auch gemeinsame Worte zu finden, die berühren (Kämmerer, 2016). Begleitende einzeltherapeutische Gespräche können erst einmal Schwierigkeiten mit den aufgeführten Aktivitäten behandeln, die Schwelle zum psychotherapeutischen Bereich reduzieren und Vertrauen für weitergehende Mitteilungen der PatientInnen schaffen.

\section{Weiterführende therapeutische Ansätze}

Da Gefühle von SSD-PatientInnen schlecht wahrgenommen oder störende körperliche Empfindungen ängstlich beobachtet und nicht als Signale für Selbstfürsorge und Selbststeuerung genutzt werden, ist die leibliche Selbstexploration immer ein zentraler Behandlungsschwerpunkt. Dafür ist es sinnvoll, die sensorische Integration anzuregen, zum Beispiel die propriozeptive Wahrnehmung der Füsse, das Gleichgewichtsempfinden mit der Arbeit auf der Balancierscheibe sowie die taktile Wahrnehmung mit strukturierter Selbstmassage zu fördern (vgl. Clauer, $2009 a+b)$, neben spielerischem Umgang mit Objekten oder auch Tanz und anderen rhythmischen Bewegungen mit oder ohne Musik. Bewegung erscheint dabei als Grundlage der Bewusstwerdung und Selbstentwicklung (Stern, 2011; Sheets-Johnson, 1999; Smith, 2010). Die grundlegende Abstimmung körperlicher Rhythmen und Erregungszustände sehen wir in der körperpsychotherapeutischen Arbeit als meist unbewusst, implizit ablaufende affektmotorische und affektsensorische Resonanzen an (vgl. Clauer, 2003; Downing, 1996; Heinrich, 2001; Heinrich-Clauer, 2008). Zudem sollen (rhythmische) Bewegungen, vor allem in einer anregenden Umgebung und Atmosphäre, die Neurogenese (Nervenzellneubildung aus Stammzellen) besonders im Bereich der Hirnregion des Hippocampus, der die Eintrittspforte für das Erlernen neuer Erfahrungsmuster ist, stimulieren (Wagner, 2007; Kempermann et al., 1998; Praag et al., 1999; vgl. Clauer, 2016, S. 128). Bewegung steigert so wohl auch beim Erwachsenen die Fähigkeit, Neues zu lernen und umzulernen. Ein Umlernen zum Beispiel der Schmerzverarbeitung ist für somatoforme Störungen höchst bedeutsam. ${ }^{1}$

Es verbessert sich zum Beispiel beim Gebrauch der Balancierscheibe neben dem Gleichgewicht auch die Integration der rechten und linken Hirnhälfte. Ballsportler bekommen dadurch eine verbesserte Koordination und treffen besser (Blakeslee \& Blakeslee, 2007), wobei in unserem Kontext eher die Verbesserung der Gefühlswahrnehmung bedeutsam ist (Lausberg, 2017).

Gesicht und Mimik verraten viel über das Innenleben eines Menschen. Neuere Forschungen zeigen dabei, dass wir auch als Erwachsene mimische Signale nur im Gesamtkontext der körperlichen Signale in ihrer Bedeutung gut entziffern können (Aviezer et al., 2012). Das bedeutet, dass die Körperbewegungen und die Signale des gesamten Körpers (Gestik) für die Abstimmungsprozesse in unseren Beziehungen wichtiger sind, als oft angenommen wird. Für unseren Kontext bedeutsam sind dabei Untersuchungsbefunde, die zeigen, dass sich die Bilder/Symbolisierung der TherapeutInnen über eine unbewusste, nonverbal gestische Kommunikation mitteilen und dies Symbolisierung und Mentalisierung bei SSD-PatientInnen fördern kann (Lausberg, 2017).

Neben den verkörperten Haltungsmustern und Organisationsprinzipien (oder Mikropraktiken) des Menschen ist die menschliche Vitalität in ihren vielfältigen Ausdruckformen einschliesslich der Dynamik des Affektgeschehens und korrigierender emotionaler Erfahrungen ein wesentlicher therapeutischer Fokus (Clauer, 2016). Wenn die Überlegungen von Stern (2011, S. 180f.) zutreffen, dass nicht Identifizierungsobjekte, sondern die Vitalitätsformen und Interaktionserfahrungen mit den Bezugspersonen internalisiert/gelernt werden, so sind die vielfältigen aktiven Methoden der HP eigentlich unverzichtbar - besonders bei SSD.

Der vitalisierende Ausdruck stimmlicher Klagen/Klagelaute in einer KPT kann sehr hilfreich sein, denn freier stimmlicher und rhythmischer Ausdruck und die damit verbundene Vertiefung der Atmung kann Vitalitätsformen aufgreifen, Zugang zu Emotionen schaffen und gehaltene Spannungen lösen (vgl. Clauer, 2016; Heinrich-Clauer, 2015). Vermutlich kommt es dabei auch zu einer Entspannung im vegetativen Nervensystem (VVC; vgl. Porges, 2010).

Bei einer Arbeit in Gruppen können Schmerzen oder körperliches Leiden äusseren Gegenständen oder Hilfsper-

1 Ein gutes Beispiel für die Fähigkeit zum Umlernen der Schmerzverarbeitung sind ÄrztInnen, die lernen (in präfrontalen Hirnregionen verankert), das Miterleben von Schmerzen ihrer PatientInnen abzuschwächen und sich so davon zu distanzieren. Dieser wohl notwendige Prozess liefert zugleich einen Erklärungsansatz für die traditionell so schwierige Kommunikation/Verständnis zwischen somatisch orientierten MedizinerInnen sowie PsychosomatikerInnen/PsychotherapeutInnen, da für letztere die Verfeinerung der Resonanzfähigkeit, der Empathie ein entscheidendes Arbeitsinstrument darstellt. 
sonen zugeschrieben (externalisiert) werden, zum Beispiel mit Techniken aus Psychodrama, Gestalttherapie, Konzentrativer Bewegungstherapie, Funktioneller Entspannung, Transaktionsanalyse. Der Dialog mit betroffenen Körperregionen ermöglicht es, sich an deren Stelle zu versetzen, deren Botschaften zu entdecken und zu einer Ich-Aussage zu machen. Aus der Position der dysfunktionalen symptomatischen Körperregion lässt sich erkunden, welche Impulse und Strebungen vom Betroffenen nicht realisiert werden und welche bisherigen Verhaltensweisen, Lebenshaltungen oder Beziehungsmuster hinderlich sind. Dann können zum Beispiel mit Psychodramatechniken oder der Technik des «leeren Stuhls» bzw. des «Inneren Dialoges» der Gestalttherapie neue Möglichkeiten herausgefunden und neue Anpassungsformen und sinnvollere Kontaktformen eingeübt werden. Auf diesem Weg lassen sich so auch soziale Probleme erkunden und soziale Fähigkeiten erlernen, wie Bedürfnisse äussern, sich selbst behaupten und Konflikte austragen.

Die Korrektur von Abstimmungsbrüchen in der Affektregulation ist ein Schlüsselprozess für die Entwicklung des Sänglings (Patientin/en). Diese Korrekturerfahrung stösst die Entwicklung interaktiver Fähigkeiten beim Kind an. Es bekommt durch erfolgreich angestossene Korrekturmassnahmen ein Gefühl für seinen Einfluss oder Kontrolle und seine Effizienz (Selbstwirksamkeit und Urheberschaft). Das Gefühl von einem eigenen Selbst (Selbstwertgefühl, Akzeptanz und Urheberschaft; vgl. Stern et al., 2012, Clauer, 2013) und die Selbstregulation werden so entscheidend gestärkt. Ein solcher Prozess setzt eine Fähigkeit zur inneren Anerkennung von eigenen Fehlern und Unzulänglichkeiten bei den Bezugspersonen (Eltern oder TherapeutInnen) voraus. Wichtig ist dabei, dass der Säugling (PatientIn) mit seinen Affekten führen kann und adäquat empathisch beantwortet wird. SSDPatientInnen sind mit einem Gefühl von Ohnmacht und Hilflosigkeit meist verzweifelt um eine Kontrolle ihrer Situation und Umwelt bemüht. Auch für Fortschritte in der Therapie ist es daher wichtig, dass PatientInnen in einem sicheren Beziehungsrahmen der Therapiesituation «führen» können. Ihre Kontrolle in der Beziehung kann Symptomatik und Schmerzerleben und damit Ohnmacht und Hilflosigkeit reduzieren (Linden, 2015). Der zuvor erwähnte Crohn-Patient war ein lehrreiches Beispiel dafür (vgl. Clauer, 2014). Weitere Therapiebeispiele können hier aus Platzgründen leider nicht ausgeführt werden.

\section{Ambulante (Einzel-)Psychotherapie}

Auch für diese Behandlungsform gelten selbstverständlich alle die zuvor ausgeführten therapeutischen Behandlungsgrundlagen. Die ambulante Therapie kann an psycho-edukative Erfahrungen anknüpfen. PatientInnen können in einer ersten Therapiephase, wenn nicht schon geschehen, Entspannungsverfahren und strukturierte körperliche Übungsprogramme erlernen, die sie dann als «Hausaufgaben» zu Hause weiter durchführen können (wie z.B. Selbstmassage, Balancierscheibe, Baumübung;
Clauer 2009a, b, 2015). Eine solche Arbeit am ganzheitlichen körperlichen Selbsterleben ermöglicht eine veränderte Wahrnehmung und Bewertung der Beschwerden und eine neue Akzeptanz für die Erkrankung (vgl. Röhricht, 2011a).

Dies führt bei einigen PatientInnen dazu, dass zuvor abgewehrte schmerzhafte Erfahrungen und Enttäuschungswut auftauchen können, für die es eine Ausdrucksmöglichkeit und ein Betrauern braucht. Dies macht dann eine intensive therapeutische Begleitung nötig.

Gefühle und Lebenshaltungen können sich in Behandlungssituationen über körperliche Resonanzprozesse, die verkörperte Gegenübertragung und unbewusst spiegelnde Imitationen übertragen. Die gemeinsamen Übungen und Erfahrungen von PatientIn und TherapeutIn schaffen einen miteinander geteilten Raum der Kooperation und so eine Grundlage für Begreifen und Benennen der Erfahrungen der/s Patientin/en. Die Verbalisierung des Erlebten durch die/den Patientin/en ist ein integraler Teil der Therapie. KPT-Ansätze haben dabei den grossen Vorteil, nicht nur berührende Worte zu nutzen, sondern auch mit achtsamer Berührung selbst arbeiten zu können. $\mathrm{Zu}$ beachtende Einschränkungen zum Beispiel in der Folge von Traumatisierungen, wurden zuvor bereits erwähnt. Berührung ist wie dargestellt auf das Innigste mit der Entstehung unseres Selbstempfindens und unserer Gefühle verbunden. Unsere Sprache bringt dies in klarer Weise zum Ausdruck: «Es hat mich etwas tief berührt.» Der von uns beschriebene Ansatz ist in diesem Sinne eine mitfühlende und sinnstiftende menschliche Begleitung von SSD-PatientInnen, die ihr Leid lindern und ihnen einen neuen Umgang damit eröffnen kann.

\section{Literatur}

Alexander, F. (1977). Psychosomatische Medizin. Grundlagen und Anwendungsgebiete. 3., unv. Aufl. Berlin: DeGruyter.

Arbeitskreis PISO (2011). PISO: Psychodynamisch-Interpersonelle Therapie bei somatoformen Störungen. Eine manualisierte Kurzzeitintervention. Göttingen: Hogrefe.

Arnim, A.v., Joraschky, P. \& Lausberg, H. (2007). Körperbild-Diagnostik. In P. Geißler \& G. Heisterkamp (Hrsg.), Psychoanalyse der Lebensbewegungen (S. 165-196). Wien: Springer.

Aviezer, H., Trope, Y. \& Todorov, A. (2012). Body Cues, not Facial Expressions Discriminate between Intense Positive and Negative Emotions. Science, 338, 1225-1229.

Ayres, A.J. (2002). Bausteine der kindlichen Entwicklung. 4. Aufl. Berlin: Springer.

Bauer J. (2011). Schmerzgrenze. Vom Ursprung alltäglicher und globaler Gewalt. 3. Aufl. München: Karl Blessing.

Beebe, B. \& Lachmann, F.M. (2004). Säuglingsforschung und die Psychotherapie Erwachsener. Stuttgart: Klett-Cotta.

Blakeslee, S. \& Blakeslee, M. (2007). The body has a mind of its own: how body maps in your brain help you do (almost) everything better. New York: Random House.

Clauer, J. (2003). Von der projektiven Identifikation zur verkörperten Gegenübertragung: Eine Psychotherapie mit Leib und Seele. Psychother. Forum, 11, 92-100.

Clauer, J. (2008). Verkörpertes (leiblich-seelisches) Begreifen: Die Behandlung psychosomatischer Erkrankungen in der Bioenergetischen Analyse. In V. Heinrich-Clauer (Hrsg.), Handbuch Bioenergetische Analyse (S. 383-409). Gießen: Psychosozial-Verlag. 
Clauer, J. (2009a). Zum Grounding-Konzept der Bioenergetischen Analyse: Neurobiologische und entwicklungspsychologische Grundlagen. Psychoanalyse \& Körper, 15(2), 79-102.

Clauer, J. (2009b). Balancierscheibe und Baumübung. In S. Fliegel \& A. Kämmerer (Hrsg.), Psychotherapeutische Schätze II (S. 29-30). Tübingen: dgvt.

Clauer, J. (2011). Die Behandlung von somatoformen Störungen in der Bioenergetischen Analyse. Sowie: Theoretische Grundlagen der Bioenergetischen Analyse - Allgemeine Einführung. In F. Röhricht (Hrsg.), Störungsspezifische Konzepte in der Körperpsychotherapie (S. 193-206, 323-332). Gießen: Psychosozial-Verlag.

Clauer, J. (2013). Die Implizite Dimension in der Psychotherapie. In P. Geißler \& A. Sassenfeld (Hrsg.), Jenseits von Sprache und Denken (S. 135-173). Gießen: Psychosozial-Verlag.

Clauer, J. (2014). Empathie als verkörperter Resonanzprozess. Von der isolierten Selbstregulation zur interaktiven Regulation. Selbstpsychologie, 52, 25-57.

Clauer, J. (2015). Verwurzelung im Körperselbst und im Primären Dreieck. Forum Bioenergetische Analyse 2015, 8-28.

Clauer, J. (2016). Sternstunden. Wirkung von Sterns Lebenswerk auf Bioenergetische Analyse (BA) und Psychoanalyse (PA). In P. Geißler (Hrsg.). Sternstunden. Daniel Sterns Lebenswerk in seiner Bedeutung für Psychoanalyse und Psychotherapie (S. 107-144). Gießen: Psychosozial-Verlag.

Damasio, A.R. (2011). Selbst ist der Mensch. Körper, Geist und die Entstehung des menschlichen Bewusstseins. München: Siedler.

Downing, G. (1996). Körper und Wort in der Psychotherapie. München: Kösel.

Downing, G. (2006). Frühkindlicher Affektaustausch und dessen Beziehung zum Körper. In G. Marlock \& H. Weiss (Hrsg.), Handbuch der Körperpsychotherapie (S. 333-361). Stuttgart: Schattauer.

Downing, G. (2007). Unbehagliche Anfänge: Wie man Psychotherapie mit schwierigen Patienten in Gang setzen kann. In P. Geißler \& G. Heisterkamp (Hrsg.), Psychoanalyse der Lebensbewegungen. Zum körperlichen Geschehen in der Psychoanalytischen Therapie - Ein Lehrbuch (S. 555-581). Wien, New York: Springer.

Eberwein, W. \& Thielen, M. (2014). Humanistische Psychotherapie. Theorien, Methoden, Wirksamkeit. Gießen: Psychosozial-Verlag.

Egger, J.W. (2017). Theorie und Praxis der Biopsychosozialen Medizin. Wien: facultas.

Eisenberger, N.I., Lieberman, M.D. \& Williams, K.D. (2003). Does rejection hurt? An fMRI Study of Social Exclusion. Science, 302, 290-292.

Eisenberger, N.I., Jarcho, J.M., Lieberman, M.D. \& Naliboff, B.D. (2006). An experimental study of shared sensitivity to physical pain and social rejection. Pain, 126, 132-138.

Ermann, M. (1997). Psychotherapeutische und psychosomatische Medizin. Stuttgart: Kohlhammer.

Fivaz-Depeursinge, E. \& Corboz-Warnery, A. (2001). Das primäre Dreieck. Heidelberg: Carl-Auer.

Fuchs, T. (2009). Leibgedächtnis und Lebensgeschichte. EXISTENZ ANALYSE, 26(2), 46-52.

Fuchs, T. \& De Jaegher, H. (2010). Understandig Intersubjectivity: Enactve and Embodied. Non-Representational Intersubjectivity. In T. Fuchs, P. Henningsen \& H. Sattel (Hrsg.), The Embodied Self (S. 203-215). Stuttgart: Schattauer.

Heinrich, V. (1986). Die Bewältigung belastender Lebensereignisse und Körperbeschwerden bei Franen. Universität Osnabrück.

Heinrich, V. (2001). Übertragungs- und Gegenübertragungsbeziehung in der Körperpsychotherapie. Psychotherapie Forum, 9, 62-70.

Heinrich-Clauer, V. (2008). Therapeuten als Resonanzkörper: Welche Saiten geraten in Schwingung. In V. Heinrich-Clauer (Hrsg.), Handbuch Bioenergetische Analyse (S. 161-178). Gießen: Psychosozial-Verlag.

Heinrich-Clauer, V. (2014). Zur Wechselwirkung von emotionaler Schutzreaktion und Muskeltonus. Sprache-Stimme - Gehör, 38, 1-6.

Heinrich-Clauer, V. (2015). Körperresonanz und Stimme. Forum Bioenergetische Analyse 2015, 29-48.
Heinrich-Clauer, V. (2017). Körperspannung als Schutzmechanismus. Eine bioenergetische Perspektive der Emotionsregulation. Psychotherapie-Wissenschaft, 7(2), 29-37.

Henningsen, P. (2007). Somatoforme Störungen als Störungen des Körperbildes. Vortrag 8. Dresdner Körperbildwerkstatt, Universitätsklinikum Carl Gustav Carus, Dresden.

Henningsen, P., Gündel, H. \& Ceballos-Baumann, A.(Hrsg.). (2006). Neuro-Psychosomatik. Grundlagen und Klinik neurologischer Psychosomatik. Stuttgart: Schattauer.

Kämmerer, W. (2016). Auf der Suche nach dem Wort, das berührt. Intersubjektivität und Fokus im psychosomatischen Dialog. Berlin: Springer.

Kempermann, G., Brandon, E. P. \& Gage, F.H. (1998). Environmental stimulation of $129 / \mathrm{SvJ}$ mice causes increased proliferation and neurogenesis in the adult dentate gyrus. Curr Biol., 8(16), 939-942.

Klitzing, K. v. (2002). Frühe Entwicklung im Längsschnitt: Von der Beziehungswelt der Eltern zur Vorstellungswelt des Kindes. Psyche, 56, 863-887.

Krause, R. (2006). Emotion, Gefühle, Affekte - Ihre Bedeutung für die seelische Regulierung. In A. Remmel, O. Kernberg, W. Vollmoeller \& B. Strauss (Hrsg.), Handbuch Körper und Persönlichkeit (S. 22-44). Stuttgart: Schattauer.

Längle, A. (2009). Das eingefleischte Selbst. EXISTENZANALYSE, 26(2), 1-34.

Lahmann, C., Allwang, C. \& Dinkel, A. (2016). Diagnostik somatoformer Störungen. Psych up2date, 10(05), 375-386. http://dx.doi. org/10.1055/s-0042-109436

LaPierre, A. (2003). From felt-sense to felt-self: Neuroaffective touch and the relational matrix. Psychologist-Psychoanalyst, 23, 1-5.

Lausberg, H. (2006). Zur Bedeutung der Kognitiven Neurowissenschaften für das Verständnis psychosomatischer Erkrankungen. In P. Hennigsen, H. Gündel \& A. Ceballos-Baumann (Hrsg.), NeuroPsychosomatik. Grundlagen und Klinik neurologischer Psychosomatik (S. 23-31). Stuttgart: Schattauer.

Lausberg, H. (2017). Körper, Körperlichkeit und Schmerz. Vortrag 19. Jahrestagung der Klinik und Poliklinik für Psychotherapie und Psychosomatik des Universitätsklinikum Carl Gustav Carus, Dresden.

Leiß, O. (2011). Ulcera duodeni und ventriculi. In R.H. Adler, W. Herzog, P. Joraschky, K. Köhle, W. Langewitz, W. Söllner \& W. Wesiak (Hrsg.), Psychosomatische Medizin. Theoretische Modelle und klinische Praxis (S. 918-929). 7., kompl. überarb. Aufl. München: Urban \& Fischer.

Lewis, R. (2008). Der Cephale Schock als somatisches Verbindungsglied zur Persönlichkeit des falschen Selbst. In V. Heinrich-Clauer (Hrsg.), Handbuch Bioenergetische Analyse (S. 113-128). Gießen: Psychosozial-Verlag.

Linden, D. J. (2015). Touch. The Science of the Sense that Makes Us Human. UK: Penguin Random House.

Morschitzky, H. (2007). Somatoforme Störungen. Diagnostik, Konzepte und Therapie bei Körpersymptomen ohne Organbefund. 2. Aufl. Wien: Springer.

Nickel, M., Cangoez, B., Bachler, E., Muehlbacher, M., Lojewski, N. \& Mueller-Rabe, N. (2006). Bioenergetic exercises in inpatient treatment of Turkish immigrants with chronic somatoform disorders: a randomized, controlled study. J Psychosom Res, 61, 507-513.

Pedrosa, G.F., Ridout, N., Kessler, H., Neuffer, M., Schoechlin, C., Traue, H.C. \& Nickel, M. (2008). Facial emotion recognition and alexithymia in adults with somatoform disorders. Depression and Anxiety, 25, 133-141.

Panagiotopoulou, E., Filippetti, M.L., Tsakiris, M. \& Fotopoulou, A. (2017). Affective touch enhances self-face recognition during multisensory integration. Scientific Reports, 7, 12883. www.nature. com/articles/s41598.017-13345-9

Porges, S. W. (2010). Die Polyvagal-Theorie. Paderborn: Junfermann.

Praag van, H., Kempermann, G. \& Gage, F.H. (1999). Running increases cell proliferation and neurogenesis in adult mouse dentate gyrus. Nat. Neurosci., 2(3), 266-270. 
Röhricht, F. (2011a). Das theoretische Modell und die therapeutischen Prinzipien/Mechanismen einer integrativen Körperpsychotherapie (KPT) bei somatoformen Störungen. Psychotherapie-Wissenschaft, 1(1), 5-13.

Röhricht, F. (Hrsg.). (2011b). Störungsspezifische Konzepte in der Körperpsychotherapie. Gießen: Psychosozial-Verlag.

Röhricht, F., Sattel, H., Kuhn, C. \& Lahmann, C. (2019). Group body psychotherapy for the treatment of somatoform disorder - a partly randomised-controlles feasibility pilot study. BMC Psychiatry, 19(1), 1-9.

Rudolf, G. (2008). Psychosomatik - konzeptuelle und psychotherapeutische Aspekte. Psychotherapie-Forum, 16, 8-14.

Sander, L. W. (2009). Die Entwicklung des Säuglings, das Werden der Person und die Entstehung des Bewusstseins. Stuttgart: Klett-Cotta.

Schaefert, R., Hausteiner-Wiehle, C., Häuser, W., Ronel, J., Herrmann, M. \& Hennigsen, P. (2012). Clinical Practice Guideline: Non-specific, functional and somatoform bodily complaints. Dtsch Arztebl Int, 109(47), 803-813.

Schnabel, U. (2019). Endlich reif im Kopf. Die Zeit, Nr. 18, S. 67.

Schore, A.N. (2005). Erkenntnisfortschritte in Neuropsychoanalyse, Bindungstheorie und Traumaforschung: Implikationen für die Selbstpsychologie. Selbstpsychologie, 6, 395-446.

Schore, A.N. (2011). The Right Brain Implicit Self lies at the Core of Psychoanalysis. Psychoanalytic Dialogues, 21, 55-74.

Sheets-Johnstone, M. (1999). The Primacy of Movement. Amsterdam: John Benjamins.

Smith, A. J. T. (2010). Comment: Minimal Conditions for the Simplest Form of Self-Consciousness. In T. Fuchs, H. C. Sattel \& P. Hennigsen (Hrsg.), The Embodied Self (S. 35-41). Stuttgart: Schattauer

Stern, D. N. (1992). Die Lebenserfahrung des Säuglings. Stuttgart: Klett-Cotta.

Stern, D. N. (2011). Ausdrucksformen der Vitalität. Frankfurt/M: Brandes \& Apsel.

Stern, D. N. et al. (The Boston Change Study Group) (2012). Veränderungsprozesse. Frankfurt/M: Brandes \& Apsel.

Traue, H. C. (1998). Emotion und Gesundheit. Die psychobiologische Regulation durch Hemmung. Heidelberg: Spektrum.

Trevarthen, C. (2009). The Intersubjective Psychobiology of Human Meaning: Learning of Culture Depends on Interest for Co-Operative Practical Work and Affection for the Joyful Art of Good Company. Psychoanalytic Dialogues, 19, 507-518.

Tschuschke, V. (2017). Psyche und Körper. Zur Psychoneuroimmunologie körperlicher Erkrankungen. Psychotherapie-Wissenschaft, 7(2), 51-62.

Uexküll, T.v., Fuchs, M., Müller-Braunschweig, H. \& Johnen, R. (Hrsg.). (1997). Subjektive Anatomie. Theorie und Praxis körperbezogener Psychotherapie. 2. Aufl. Stuttgart: Schattauer.

Wagner, P. (2007). Nervenkitzel. Cavallo, 11, 45-49.

\section{Somatic symptom disorders in Humanistic and Body Psychotherapy}

Abstract: Meeting patients with «somatic symptom disorders» our leading impression will be their complaint about only bodily symptoms with a strict refusal of psychosomatic explanations. Those patients are mostly seen as «difficult». They experience pain only on the physical level without access to an emotional and psychic context in relationships (alexithymia). Some etiologic considerations are helpful to develop a deeper comprehension about their specific ways of suffering from painful physical and relational distress. SSD-patients mostly didn't experienced a loving embodied emotional and psychic resonance with their primary caretakers. By the way they were handled in their own body they learned to consider their body as an object of dysfunction and hostility. Body Psychotherapy easily can pick-up their focus of symptoms. This work presents principles and possibilities to help patients to improve their body-awareness as fundamental basis for helpful changes. As far as possible the developmental and neurobiological context and background is presented. The patients then do develop more agency and self-confidence in the way of handling their diseases by the newly acquired (bodily) self-awareness and educational information. In addition they evolve new possibilities to organize their life and relationships in more functional and confident ways. Some research studies support that the presented forms and principles of treatment can be helpful for SSD-patients.

Key words: Somatoform symptom disorder, pain, alexithymia, disorder of body schema, family cooperation, body psychotherapy, training of body-awareness

\section{Disturbi Somatoformi nella Psicoterapia umanistica e corporea}

Riassunto: I pazienti considerati difficili con disturbi Somatoformi (SSD) si rivolgono a noi con lamentele relative esclusivamente a disturbi fisici ed un rigoroso rifiuto di spiegazioni psicosomatiche. L'esperienza del dolore, ad esempio, è percepita solo fisicamente e non trovano un contesto di relazione psico-emotiva (alessitimia). Le considerazioni eziologiche ci aiutano a comprendere meglio la loro esperienza fisica dolorosa specifica e le loro forme di relazione. I pazienti con SSD di solito non hanno sperimentato alcuna risonanza affettiva, fisico-emotiva amorevole nelle prime figure di riferimento e hanno imparato a considerare i loro corpi come oggetti (disfunzionali). La psicoterapia corporea può comprendere bene questo focus sui sintomi. Vengono descritti i principi e le possibilità di una promozione differenziata della percezione corporea come base per utili cambiamenti nell'SSD e, se disponibili, vengono spiegate correlazioni di psicologia dello sviluppo e di neurobiologia. La nuova consapevolezza di sé e le informazioni esplicative aiutano i pazienti ad essere più autodeterminati/più efficaci nell'affrontare i loro disturbi e a trovare nuove possibilità di usare i sentimenti per modellare le relazioni e le loro vite. Gli studi esistenti suggeriscono che una procedura con i principi di base qui descritti può essere utile.

Parole chiave: disturbo Somatoforme, esperienza del dolore, alessitimia, disturbo dello schema corporeo, collaborazione familiare, psicoterapia corporea, formazione per la percezione corporea

\section{Der Autor}

Jörg Clauer, Dr. med., Dipl. Biochemiker, ist Arzt für Psychosomatische Medizin und Psychoanalyse, Psychiatrie und Psychotherapie, Allgemeinmedizin und Rehabilitationswesen, seit 1999 in eigener Praxis in Osnabrück, zuvor als Leiter von psychosomatischen Fachkliniken tätig. Er ist Dozent, Lehrtherapeut und Lehrsupervisor für Psychotherapie, Bioenergetische Analyse, Psychoanalyse und Psychodrama sowie internationaler Trainer für Bioenergetische Analyse (IIBA Faculty).

\section{Kontakt}

Dr. Jörg Clauer

Krahnstr. 17, D-49074 Osnabrück

Tel.: +495412023100

E-Mail: joerg.clauer@osnanet.de 